\title{
Commercial Availability of Predatory Mites ${ }^{1}$
}

\author{
Silvia I. Rondon, James F. Price, Oscar E. Liburd, Roger Francis, Daniel J. Cantliffe ${ }^{2}$
}

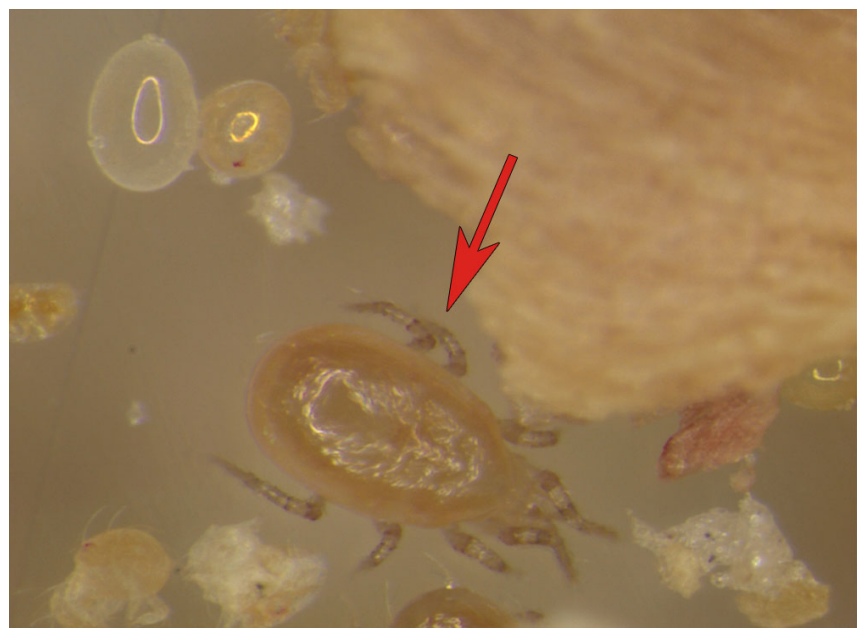

Figure 1. Adult of Neoseiulus californicus $(0.1 \mathrm{~mm}$ in length $\times 0.06 \mathrm{~mm}$ in width). Credits: E. Jovicich, UF/IFAS

Predatory mites such as Neoseiulus californicus McGregor (Fig. 1) and Phytoseiulus persimilis Athias-Henriot (Fig. 2) feed on important fruits and ornamental pests such as the twospotted spider mite (Tetranychus urticae Koch), broad mite (Polyphagotarsonemus (Stenotarsonemus) latus Banks), cyclamen mite (Tarsonemus pallidus L.), and other mite species. These predatory mites are associated with several agricultural cropping systems including strawberries, raspberries, roses, grapes, citrus, ornamentals, and vegetables. Predatory mites have been used as an alternative to miticides on a variety of crops. Their commercial availability is crucial.

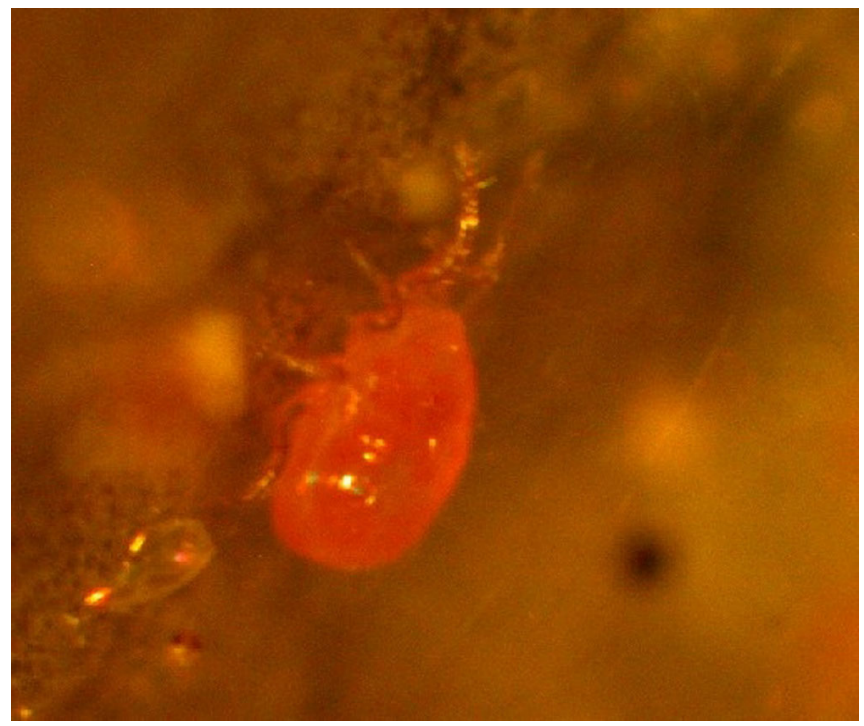

Figure 2. Adult of Phytoseiulus persimilis $(0.2 \mathrm{~mm}$ in length $\mathrm{X} 0.08 \mathrm{~mm}$ in width). Credits: E. Jovicich, UF/IFAS

Listed below are suppliers of predatory mites with their names, addresses, and telephone and fax numbers included. Target pests must be identified correctly before selecting a control agent, and an

1. This document is HS1000, one of a series of the Horticultural Sciences Department, Florida Cooperative Extension Service, Institute of Food and Agricultural Sciences, University of Florida. Publication date: November 2004. Please visit the EDIS Web site at http://edis.ifas.ufl.edu.

2. Silvia I. Rondon, adjunct research associate, Horticultural Sciences Department; James F. Price, associate professor, GCREC-Bradenton; Oscar E. Liburd, assistant professor, Entomology and Nematology Department; Roger Francis, Clemson Extension Service, St. Charleston, South Carolina; Daniel J. Cantliffe, professor and chair, Horticultural Sciences Department, Cooperative Extension Service, Institute of Food and Agricultural Sciences, University of Florida, Gainesville, 32611.

The Institute of Food and Agricultural Sciences (IFAS) is an Equal Employment Opportunity - Affirmative Action Employer authorized to provide research, educational information and other services only to individuals and institutions that function without regard to race, creed, color, religion, age, disability, sex, sexual orientation, marital status, national origin, political opinions or affiliations. For information on obtaining other extension publications, contact your county Cooperative Extension Service office. Florida Cooperative Extension Service / Institute of Food and Agricultural Sciences / University of Florida / Larry R. Arrington, Interim Dean 
assurance of vigor of the shipped predatory mites

must be established before releasing the agents. For more information, visit

strawberry.ifas.ufl.edu/BerryTimes/BVTDec03.htm.

\section{Suppliers of Predatory Mites in the USA and Canada}

\author{
Associates Insectary \\ P.O. Box 969 \\ Santa Paula, CA 93061-0969 \\ Tel: (805) 933-1301 \\ Fax: (805) 933-1304 \\ E-mail: info@associatesinsectary.com \\ Web: www.associatesinsectary.com
}

\section{Biotactics (Benemite)}

20780 Warren Road

Perris, CA 92570

Tel: (909) 943-2819

E-mail: sales@benemite.com

Web: www.benemite.com

\section{Bio Ag Services}

4218 W Muscat

Fresno, CA 93706

Tel: (559) 268-2835

E-mail: bioag@theworks.com

\section{Biobest}

2020 Mersea Road \#3 RR4

Leamington, Canada, 0N N8H 3 V7

Tel: (519) 322-2178

E-mail: biobest@on.aibn.com

Web: www.biobest.be

Biofac, Inc.

P.O. Box 87

Mathis, TX 78368

Tel: (800) 233-4914

\section{Buena Biosystems}

P.O. Box 4008

Ventura, CA 93007-7760

Tel: (805) 525-2525

Fax: (805) 525-6058

E-mail: bugdude@msn.com

\section{Central Coast Insectary}

313 Hames Road

Watsonville, CA 95076

Tel: (408) 726-1853

Ecosolutions, Inc.

2948 Landmark Way

Palm Harbor, FL 34684

Tel/Fax: (727) 787-3669

E-mail: ecosolutions@mindspring.com

The Green Spot, Ltd.

93 Priest Road

Nottingham, NH 03290-6204

Tel: (603) 942-8925

E-mail: info@greenmethods.com

Web: www.greenmethods.com

\section{Harmony Farm Supply}

P.O. Box 460

Graton, CA 95444

Tel: (707) 823-9125

E-mail: info@harmonyfarm.com

Hydro-Gardens, Inc.

P. O. Box 9707

Colorado Springs, CO 80932

Tel: (800) 634-6362

IPM Laboratories, Inc.

980 Main Street

Locke, NY 13092-0300

Tel: (315) 497-2063

Fax: (315) 497-3129

E-mail: ipmlabs@baldcom.net

Web: www.ipmlabs.com

\section{Koppert Biological Systems}

28465 Beverly Road

Romulus, MI 48174

E-mail: asktheexpert@ koppertonline.com

Web: www.koppertonline.com

Ladybug Sales

P.O. Box 903

Gridley, CA 95948 
Tel: (916) 868-1627

\section{M \& R Durango, Inc.}

6565 Highway 172

Ignacio, CO 81137

Tel: (800) 526-4075 or (970) 259-3521

Fax: (970) 259-3857

E-mail: mrdrango@frontier.net

Web: www.goodbug.com

\section{Natural Insect Control}

R.R. \# 2 Stevensville

Ontario, Canada, LOS 1S0

Tel: (905) 382-2904

Fax: (905) 382-4418

E-mail: nic@ niagara.com

Web: www.natural-insect-control.com

\section{Natural Pest Controls}

8864 Little Creek Drive

Orangeville, CA 95662

Tel: (916) 726-0855 or (916) 923-3353

Fax: (916) 923-1278

Necessary Trading Co.

P.O. Box 603

New Castle, VA 24127

Tel: (703) 864-5103

New Earth, Inc.

4422 E Highway 44

Shepardsville, KY 40165

Tel: (502) 543-5933

\section{Pacific Tree Farms}

4301 Lynwood Drive

Chula Vista, CA 92010

Tel: (619) 422-2400

\section{Peaceful Valley Farm Supply}

P.O. Box 2209

Grass Valley, CA 95945

Tel: (888) 784-1722 or (530) 272-4769

Web: www.groworganic.com

Plant Sciences, Inc. / Koppert

342 Green Valley Road
Watsonville, CA 95076

Tel: (831) 728-7771

E-mail: tkueneman@plantsciences.com

\section{PRAXIS}

P.O. Box 360

2723 116th Ave.

Allegan, MI 49010

Tel: (269) 673-2793

Sierra Ag

2749 E Malaga

Fresno, CA 93725

Tel: (209) 233-0585

\section{Sterling Insectary}

P.O. Box 1987

Delano, CA 93216

Tel: (661) 725-1832

E-mail:matt@sterlingnursery.com

Worm's Way, Inc.

7850 North State Road 37

Bloomington, IN 47404

Tel: (800) 274-9676 or (812) 876-6450

Fax: (800) 316-1264

E-mail: chrisct@wormsway.com

Web: www.wormsway.com

Note: These suppliers of beneficials are presented for information only and are not endorsed by the authors. 